\title{
Terahertz and infrared spectroscopic evidence of phonon-paramagnon coupling in hexagonal piezomagnetic $\mathrm{YMnO}_{3}$
}

\author{
C. Kadlec, ${ }^{1}$ V. Goian, ${ }^{1}$ K. Z. Rushchanskii, ${ }^{2}$ P. Kužel, ${ }^{1}$ M. Ležaić,,${ }^{2}$ K. Kohn,${ }^{3}$ R. V. Pisarev, ${ }^{4}$ and S. Kamba ${ }^{1, *}$ \\ ${ }^{1}$ Institute of Physics ASCR, Na Slovance 2, 18221 Prague 8, Czech Republic \\ ${ }^{2}$ Peter Grünberg Institut, Forschungszentrum Jülich GmbH, 52425 Jülich and JARA-FIT, Germany \\ ${ }^{3}$ Waseda University, Department of Physics, Tokyo 169-8555, Japan \\ ${ }^{4}$ Ioffe Physical-Technical Institute, Russian Academy of Sciences, 194021 St. Petersburg, Russia
}

(Received 31 August 2011; revised manuscript received 4 November 2011; published 22 November 2011)

\begin{abstract}
Terahertz and far-infrared electric and magnetic responses of hexagonal piezomagnetic $\mathrm{YMnO}_{3}$ single crystals are investigated. Antiferromagnetic resonance is observed in the spectra of magnetic permeability $\mu_{a}[\mathbf{H}(\omega)$ oriented within the hexagonal plane] below the Néel temperature $T_{N}$. This excitation softens from 41 to $32 \mathrm{~cm}^{-1}$ upon heating and finally disappears above $T_{N}$. An additional weak and heavily-damped excitation is seen in the spectra of complex dielectric permittivity $\varepsilon_{c}$ within the same frequency range. This excitation contributes to the dielectric spectra in both antiferromagnetic and paramagnetic phases. Its oscillator strength significantly increases upon heating toward room temperature, thus providing evidence of piezomagnetic or higher-order couplings to polar phonons. Other heavily-damped dielectric excitations are detected near 100 $\mathrm{cm}^{-1}$ in the paramagnetic phase in both $\varepsilon_{c}$ and $\varepsilon_{a}$ spectra, and they exhibit similar temperature behavior. These excitations appearing in the frequency range of magnon branches well below polar phonons could remind electromagnons, however their temperature dependence is quite different. We have used density functional theory for calculating phonon dispersion branches in the whole Brillouin zone. A detailed analysis of these results and of previously published magnon dispersion branches brought us to the conclusion that the observed absorption bands stem from phonon-phonon and phonon-paramagnon differential absorption processes. The latter is enabled by strong short-range in-plane spin correlations in the paramagnetic phase.
\end{abstract}

PACS number(s): 75.85.+t, 78.30.-j, 63.20.D-, 75.30.Ds

\section{INTRODUCTION}

Spin waves (magnons) in magnetically ordered materials can be excited by the magnetic component $\mathbf{H}(\omega)$ of the electromagnetic radiation, giving rise to a resonant dispersion of magnetic permeability in the microwave or terahertz (THz) frequency region. Recently, new coupled spin-lattice excitations named electromagnons have been discovered in multiferroics, where the magnetic order coexists with the ferroelectric one. ${ }^{1,2}$ Electromagnons are excited by the electric component $\mathbf{E}(\omega)$ of the electromagnetic radiation, therefore they can be detected in the $\mathrm{THz}$ dielectric permittivity spectra. Though they were theoretically predicted in $1970,{ }^{3}$ the first experimental confirmation appeared in 2006. ${ }^{1}$ These excitations were mainly investigated in the rare earth $(R)$ orthorhombic manganites $R \mathrm{MnO}_{3}$ and $R \mathrm{Mn}_{2} \mathrm{O}_{5}$ (for reviews see, e.g., Refs. 4-6), and in hexaferrites. ${ }^{7}$

Multiferroics can be roughly divided into two groups. ${ }^{8-10} \mathrm{In}$ the so-called type-I multiferroics, the ferroelectric (FE) order takes place both above and below the magnetic ordering temperature, and the spontaneous polarization is large. However, the coupling between magnetic and electric order parameters is weak.

A general feature of type-II multiferroic materials is that the ferroelectric phase is induced by magnetic ordering characterized by a particular type of incommensurate spiral magnetic structure. In this case, the magnetically-induced polarization is by several orders of magnitude smaller than in type-I multiferroics. However the coupling between electric and magnetic subsystems is large, and giant magnetoelectric effects are observed. The magnon dispersion branch in the incommensurate phase exhibits a minimum at the wave vector $\boldsymbol{q}_{m}$ corresponding to the modulation vector of the ordered spins. In contrast to the magnetic resonance (magnon at $\mathbf{q} \approx 0$ ) characterized by sharp spectral features, the electromagnons manifest themselves as very broad spectral bands, because their activation in the dielectric spectra is closely related to the high density of states close to the extrema of the magnon dispersion branches. Since the probing $\mathrm{THz}$ radiation has a long wavelength (i.e., the wave vector $\mathbf{q} \approx 0$ ), the electromagnons cannot be excited by a resonant single-photon absorption due to the wave vector conservation law; in this sense polar phonons should be involved in the interaction process.

An experimentally observed low-frequency electromagnon in type-II multiferroics was found to be related to the spin waves near the magnetic Brillouin zone (BZ) center with $\mathbf{q}=\mathbf{q}_{m}{ }^{4,11}$ or to those with $\mathbf{q}=\mathbf{q}_{\mathrm{BZE}}-2 \mathbf{q}_{m},{ }^{12}$ here $\boldsymbol{q}_{\mathrm{BZE}}$ stands for the wave vector at the BZ edge. In both cases, the low-frequency electromagnon has a similar frequency as the magnon with $q=0$, which is expected because all these excitations are related to the same magnon branch. A high-frequency electromagnon corresponds to an excitation of the BZ-edge magnons $\left(\mathbf{q}=\mathbf{q}_{\mathrm{BZE}}\right)$ which can induce a quasiuniform modulation $(\mathbf{q} \approx 0)$ of the local electric dipole moment. ${ }^{11,13}$ As for mechanisms of the electromagnon excitations, some researchers claim that the low-frequency electromagnons are activated by the inverse DzyaloshinskiiMoriya mechanism, while the high-frequency one is activated by the Heisenberg exchange coupling. ${ }^{5,6}$ Other authors believe that both types of electromagnons can be explained by the Heisenberg exchange coupling. ${ }^{14}$

Formerly, it was assumed that the electromagnons can be activated only in type-II multiferroics due to the large 
magnetoelectric coupling. Nevertheless, electromagnons were recently observed also in $\mathrm{BiFeO}_{3},{ }^{15-17}$ which is the most prominent type-I multiferroic with a rather weak magnetoelectric coupling. In this context, $\mathrm{THz}$ dielectric spectra of multiferroics may shed new light on the nature of magnetoelectric coupling.

Hexagonal manganites $R \mathrm{MnO}_{3}$ belong to the type-I multiferroics. In particular, the hexagonal $\mathrm{YMnO}_{3}$ is ferroelectric below $\approx 1250 \mathrm{~K}^{18}$ and the antiferromagnetic (AFM) ordering sets only below $\mathrm{T}_{N} \approx 70 \mathrm{~K} \cdot{ }^{19,20}$ The magnetic symmetry is $P \underline{6}_{3} \mathrm{~cm}^{21}$ and therefore the linear magnetoelectric coupling is forbidden. However, piezomagnetic and magnetoelastic couplings and higher-order magnetoelectric couplings are allowed. ${ }^{22-24}$ The piezomagnetic coupling is characterized by a bilinear interaction between the magnetic order parameter and strain, in contrast to the magnetoelastic coupling which is proportional to the product of squared order parameter and strain. $^{22,25}$ By using the method of optical second harmonic generation, ${ }^{26}$ the piezomagnetic coupling was observed owing to the interaction between AFM and FE domain walls in $\mathrm{YMnO}_{3} \cdot{ }^{23,24}$ Switching of the $\mathrm{FE}$ polarization triggers a reversal of the AFM order parameter. ${ }^{23,24,27}$ Higher order magnetoelectric coupling in $\mathrm{YMnO}_{3}$ has been observed in several works. Exceptionally large atomic displacements at $T_{N}$ were observed in structural studies, and they demonstrate unusually strong magnetoelastic coupling. ${ }^{28}$ The large spinpolar-phonon coupling manifests itself by a decrease of the low-frequency permittivity ${ }^{29}$ near $T_{H}$, which is probably caused by anomalous hardening of several infrared-active phonons. ${ }^{30}$ Similar phonon anomalies were observed near $T_{N}$ also in the Raman spectra. ${ }^{31}$ Ultrasound measurements on a single crystal of the hexagonal $\mathrm{YMnO}_{3}$ showed anomalous behavior of the elastic moduli $C_{11}$ and $C_{66}$ due to a strong coupling of the lattice with the in-plane exchange interaction. ${ }^{32}$

The AFM resonance in hexagonal $\mathrm{YMnO}_{3}$ crystal was first reported in Ref. 33. More detailed $\mathrm{THz}$ studies of $\mathrm{YMnO}_{3}$ ceramics were recently published in Ref. 34. The AFM resonance lies near $43 \mathrm{~cm}^{-1}$ at $4 \mathrm{~K}$, and its frequency softens upon heating toward $T_{N}$, where it disappears. ${ }^{33,34}$ Three magnon branches were discovered below $T_{N}$ using inelastic neutron scattering (INS). ${ }^{20,35,36}$ Two of them are degenerated near the BZ center, and their frequencies correspond to the above-mentioned AFM resonance. Moreover, a possible existence of magnons and short-range correlations between spins at Mn sites in paramagnetic phase were indicated by INS. ${ }^{37-39}$ The magnetoelastic coupling manifests itself also by a strong mixing of magnons with acoustic phonons; this leads to a gap in the transverse acoustic (TA) phonon branch occurring at the frequencies and wave vectors where the uncoupled magnon and TA branches would intersect. ${ }^{36}$ Recent polarized INS measurements revealed that the excitation detected at liquid helium temperatures near $43 \mathrm{~cm}^{-1}$ has a mixed character of magnetic spin wave and lattice vibration, ${ }^{40}$ i.e., its contribution to both the magnetic permeability and the dielectric permittivity is possible.

The reported piezomagnetic, magnetoelastic, and higherorder magnetoelectric couplings in optical, acoustic, and mainly INS data stimulated our spectroscopic study of hexagonal single crystals of $\mathrm{YMnO}_{3}$. In this paper, we present results on far-infrared (FIR) and $\mathrm{THz}$ polarized spectra in this material emphasizing interaction between magnetic, electric, and phonon subsystems. We demonstrate that strongly underdamped AFM resonance observed near $\approx 40 \mathrm{~cm}^{-1}$ contributes only to the magnetic permeability spectra below $T_{N}$. An additional broad and weak absorption band was observed in the same frequency range in the dielectric spectra both below and above $T_{N}$. In contrast to electromagnons which are typically observed only below $50 \mathrm{~K}$, the oscillator strength of this excitation significantly increases upon heating when room temperature is approached. This indicates that the feature must be related to the occupation number of magnons and/or phonons. An additional absorption band with similar temperature behavior was observed also near $100 \mathrm{~cm}^{-1}$. We will show that both these excitations can be explained by differential multiphonon and magnon-phonon processes.

\section{EXPERIMENTAL DETAILS}

The experiments were performed using a Fourier-transform infrared (FTIR) spectrometer Bruker IFS $113 \mathrm{v}$ and a custom-made $\mathrm{THz}$ time-domain spectrometer. ${ }^{41}$ In both experiments, Optistat CF cryostats (Oxford Instruments) with polyethylene (FIR) or Mylar (THz) windows were used for measurements between 10 and $300 \mathrm{~K}$. A helium-cooled bolometer operating at $1.6 \mathrm{~K}$ was used as a detector in the FTIR spectrometer. Principles of $\mathrm{THz}$ time-domain spectroscopy are explained in Ref. 42. The output of a femtosecond Ti:sapphire laser oscillator (Coherent, Mira) excites an interdigitated photoconducting switch TeraSED (Giga-Optics) to generate linearly polarized broadband $\mathrm{THz}$ probing pulses. A gated detection scheme based on an electro-optic sampling with a 1-mm-thick [110] ZnTe crystal permits to measure the time profile of the electric field of the transmitted $\mathrm{THz}$ pulse (see Ref. 41 for further details).

Hexagonal $\mathrm{YMnO}_{3}$ single crystals were grown by the floating zone method. ${ }^{43}$ Two crystal plates with lateral dimensions of $\sim 4.5 \times 5 \mathrm{~mm}^{2}$ and with the $c$ axis oriented either in-plane or out-of-plane along its normal, were cut and polished to obtain highly plane-parallel samples (within $\pm 1 \mu \mathrm{m}$ ) with thicknesses of 1100 and $348 \mu \mathrm{m}$ for each orientation, respectively. These crystal plates were probed using the $\mathrm{THz}$ and FIR beam in all possible geometries: $\mathbf{E}(\omega) \perp \mathbf{c}, \mathbf{H}(\omega) \perp \mathbf{c} ; \mathbf{E}(\omega) \perp \mathbf{c}, \mathbf{H}(\omega) \| \mathbf{c} ;$ and $\mathbf{E}(\omega) \| \mathbf{c}, \mathbf{H}(\omega) \perp$ c. It enabled us to get access to the complex spectra of the products $\varepsilon_{a} \mu_{a}, \varepsilon_{a} \mu_{c}$, and $\varepsilon_{c} \mu_{a}$ as shown in Figs. 1(a), 1(b), and $1(\mathrm{c})$, respectively.

\section{RESULTS}

At low temperatures, the peak around $40 \mathrm{~cm}^{-1}$ seen in the spectra of $\varepsilon_{a} \mu_{a}$ and $\varepsilon_{c} \mu_{a}$ [Figs. 1(a) and 1(c)] but not in those of $\varepsilon_{a} \mu_{c}$ [Fig. 1(b)] is definitely due to the AFM resonance as it contributes only to the magnetic permeability $\mu_{a}$. The AFM resonance vanishes above $T_{N} \sim 70 \mathrm{~K}$. The data shown in Fig. 1(b) allow us to assume that $\mu_{c}=1$ in the $\mathrm{THz}$ range. This is in agreement with the magnetic order of $\mathrm{YMnO}_{3}$ in the AFM phase: The spins are ordered in adjacent layers in the hexagonal plane in such a way that the magnetic resonances are not expected to be excited with $\mathbf{H} \| \mathbf{c}$. Based on this assumption, we are able to retrieve the complex values of the permeability $\mu_{a}$ and of the permittivity $\varepsilon_{c}$ (see Fig. 2). 


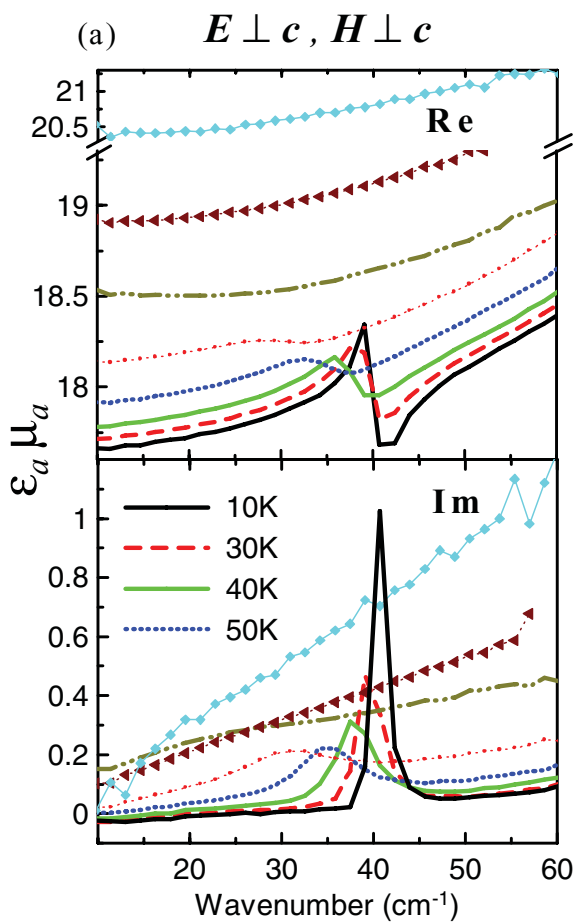

(b)

$\boldsymbol{E} \perp \boldsymbol{c}, \boldsymbol{H} / / \boldsymbol{c}$

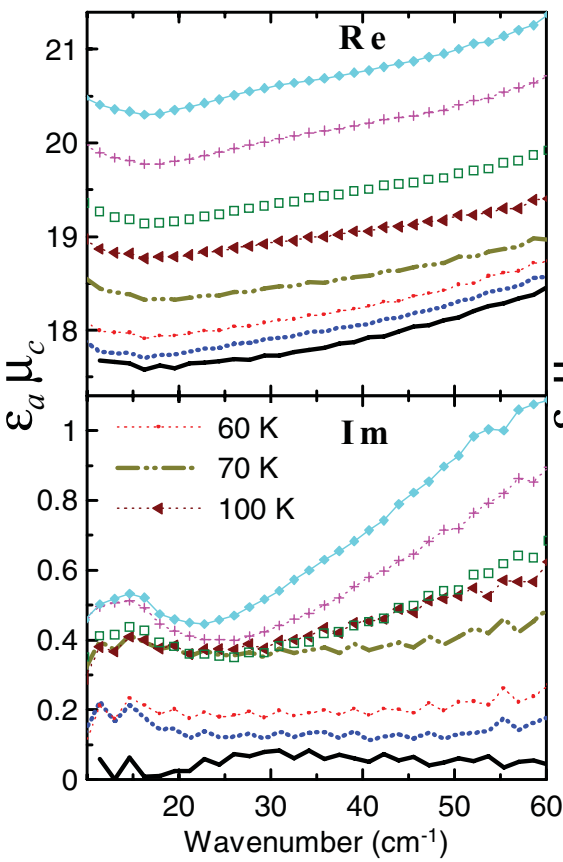

(c)

$E / / c, H \perp c$

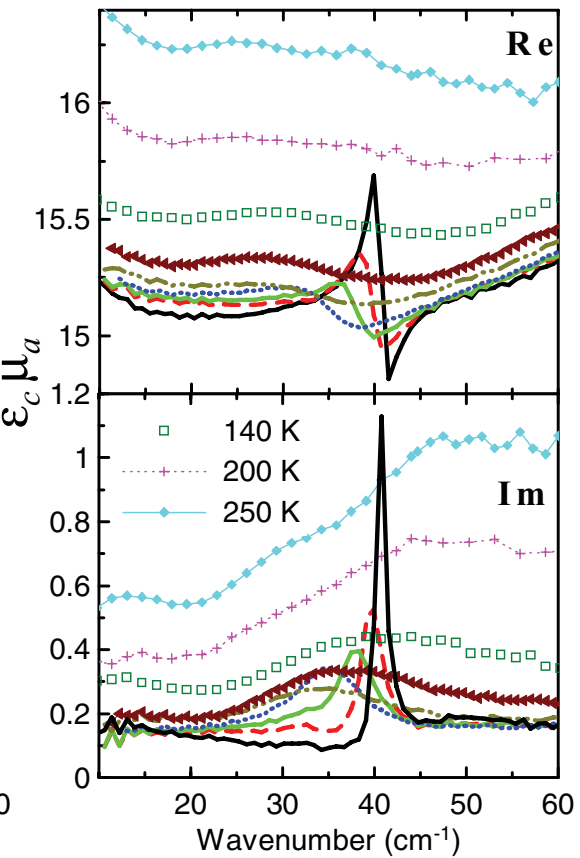

FIG. 1. (Color online) Complex THz spectra of $\mathrm{YMnO}_{3}$ taken at various temperatures. The polarization of the THz beam is indicated above the plots. The resonance feature near $\sim 40 \mathrm{~cm}^{-1}$ corresponds to the doubly-degenerated AFM mode contributing to the magnetic permeability $\mu_{a}$ spectra.

The spectra of $\mu_{a}$ were fitted by a damped harmonic oscillator, and the resulting AFM resonance frequency is plotted in Fig. 3; a strong softening is observed upon heating toward $T_{N}$. Similar temperature dependence was briefly published earlier, ${ }^{33,34}$ with the magnon frequency higher by approximately $2 \mathrm{~cm}^{-1}$. Besides the sharp AFM resonance line in the low-temperature $\mu_{a}$ spectra, one can observe a broad dielectric absorption band around $40 \mathrm{~cm}^{-1}$ in the $\varepsilon_{c}$ spectra. This feature is detected even above $T_{N}$, where its strength remarkably increases with temperature. The presence

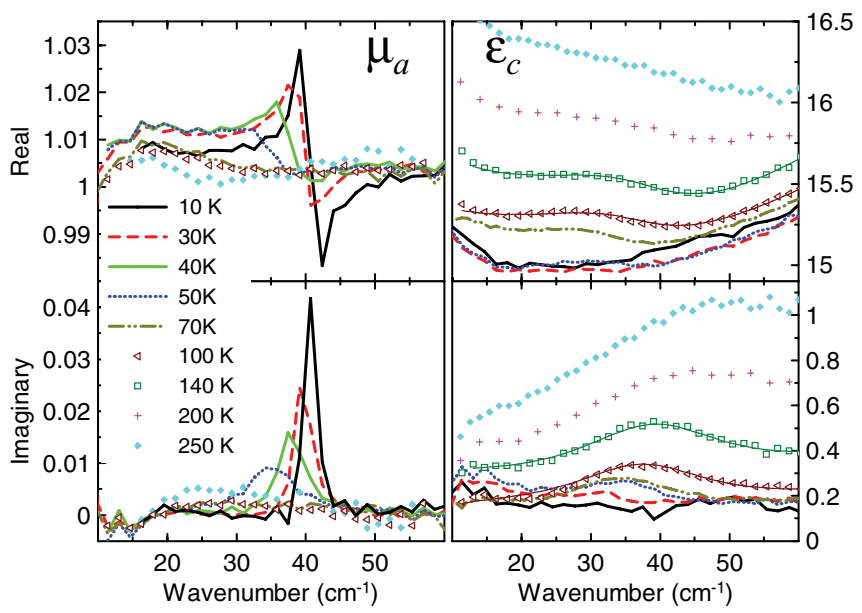

FIG. 2. (Color online) Temperature dependence of the complex permittivity $\varepsilon_{c}$ and permeability $\mu_{a}$ spectra calculated from data plotted in Fig. 1. The solid $\varepsilon_{c}$ curves at 100 and $140 \mathrm{~K}$ result from the oscillator fit. of such a resonance in $\varepsilon_{c}$ is qualitatively expected from a simple comparison of the raw data in Figs. 1(a) and 1(c). The accessible spectral range of the $\mathrm{THz}$ measurements for our sample is limited to $\sim 60 \mathrm{~cm}^{-1}$, therefore we have performed also FTIR transmission (up to $100 \mathrm{~cm}^{-1}$ ) and reflectivity (up to $650 \mathrm{~cm}^{-1}$ ) measurements for all polarizations.

An example of FTIR experimental transmittance and reflectivity spectra obtained at $120 \mathrm{~K}$ and their various

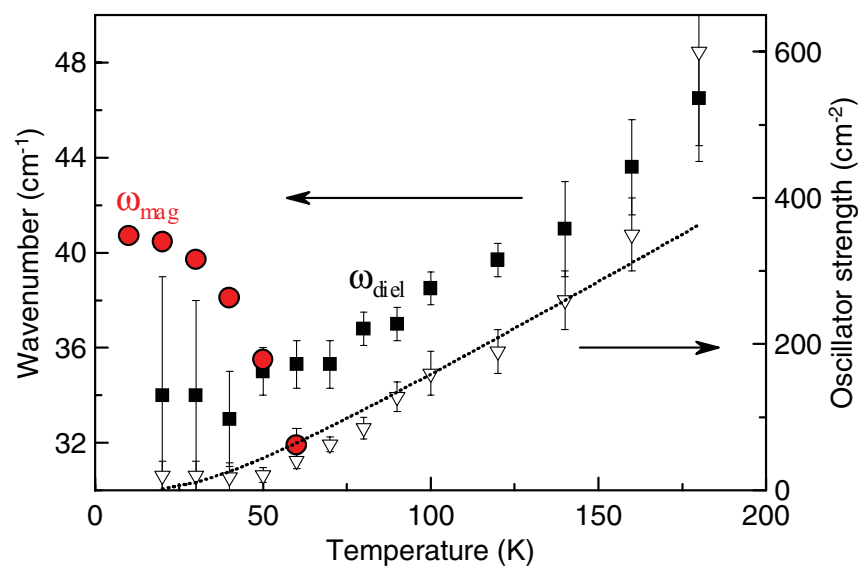

FIG. 3. (Color online) Temperature dependences of parameters of the resonances observed in magnetic $\mu_{a}$ and dielectric $\varepsilon_{c}$ spectra. Closed circles: frequency of the AFM resonance. Solid squares and open triangles: eigenfrequency $\omega_{\text {diel1 }}$ and oscillator strength $\Delta \varepsilon \omega_{\text {diel1 }}^{2}$, respectively, of the mode observed in the dielectric spectra in Fig. 2. The dotted line shows the population increase of an energy level at $66 \mathrm{~cm}^{-1}$ following the Bose-Einstein statistics. 


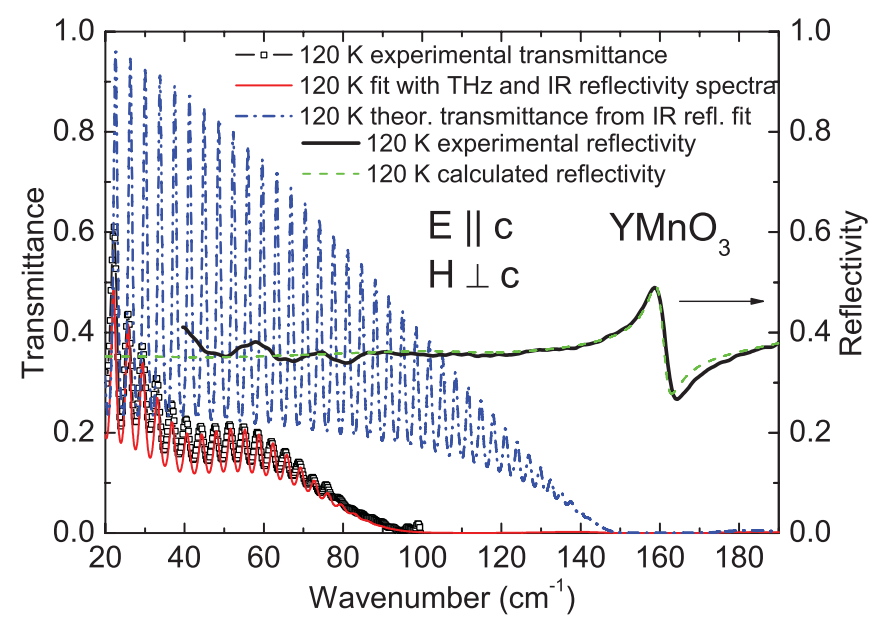

FIG. 4. (Color online) Example of the experimental FTIR transmittance and reflectivity spectra of $348-\mu$ m-thick $\mathrm{YMnO}_{3}$ crystal with polarization $\mathbf{E} \| \mathbf{c}$ obtained at $120 \mathrm{~K}$. Dashed-dotted blue line: theoretical transmittance spectrum obtained from parameters of the FTIR reflectivity fit (without considering modes observed by $\mathrm{THz}$ spectroscopy); solid red line: simultaneous fit of the FTIR transmission and THz spectra. Dashed green line is the result of a fit of the reflectivity using the parameters obtained from the fit of FIR and $\mathrm{THz}$ transmittance. One can see that the reflectivity spectrum is not sensitive enough to detect the weak broad modes near 40 and $100 \mathrm{~cm}^{-1}$. Oscillations in the experimental reflectivity spectrum observed below $80 \mathrm{~cm}^{-1}$ are caused by the diffraction of FIR beam on a small sample.

fits are shown in Fig. 4. Regular oscillations observed in the transmittance spectrum are due to Fabry-Pérot interferences in the plane-parallel sample; a weak minimum near $40 \mathrm{~cm}^{-1}$ corresponds to the broad absorption band detected in the THz dielectric spectra (see Fig. 2). According to Ref. 30 as well as according to our FTIR reflectivity (see e.g. Fig. 4), the lowest frequency polar phonons lie above $150 \mathrm{~cm}^{-1}$ in both polarized $\mathbf{E} \| \mathbf{c}$ and $\mathbf{E} \perp \mathbf{c}$ spectra. Nevertheless, our simultaneous fits of the THz complex permittivity and FTIR transmittance and reflectivity data reveal several additional modes below these phonon frequencies. The relevant spectra are plotted in Fig. 5. Besides the sharp magnon line at $40 \mathrm{~cm}^{-1}$, three other broad modes at roughly 10,40 , and $100 \mathrm{~cm}^{-1}$ were used in the fitting procedure in order to account for the measured shape of the $\mathbf{E} \| \mathbf{c}$ spectra at $10 \mathrm{~K}$ [see Fig. 5(a)]. The additional modes remain in the spectra up to room temperature and their strength increases upon heating. Also in $\mathbf{E} \perp \mathbf{c}$ polarized spectra, two broad modes observed near 10 and $90 \mathrm{~cm}^{-1}$ were used for the fits above $50 \mathrm{~K}$.

The feature observed near $10 \mathrm{~cm}^{-1}$ in both polarized spectra could be related to low-frequency magnons ${ }^{35}$ (cf., the low-frequency magnon branches shown in Fig. 7). However, the sensitivity and accuracy of our $\mathrm{THz}$ spectra below 20 $\mathrm{cm}^{-1}$ is limited; therefore we cannot exclude that it is only an artifact. For this reason, we will not speculate about the origin of this excitation. All other modes appearing below $150 \mathrm{~cm}^{-1}$ are clearly observed in the $\mathrm{THz}$ and/or FTIR transmittance spectra, while the FTIR reflectivity measurements are not sensitive enough to detect and resolve these weak and broad

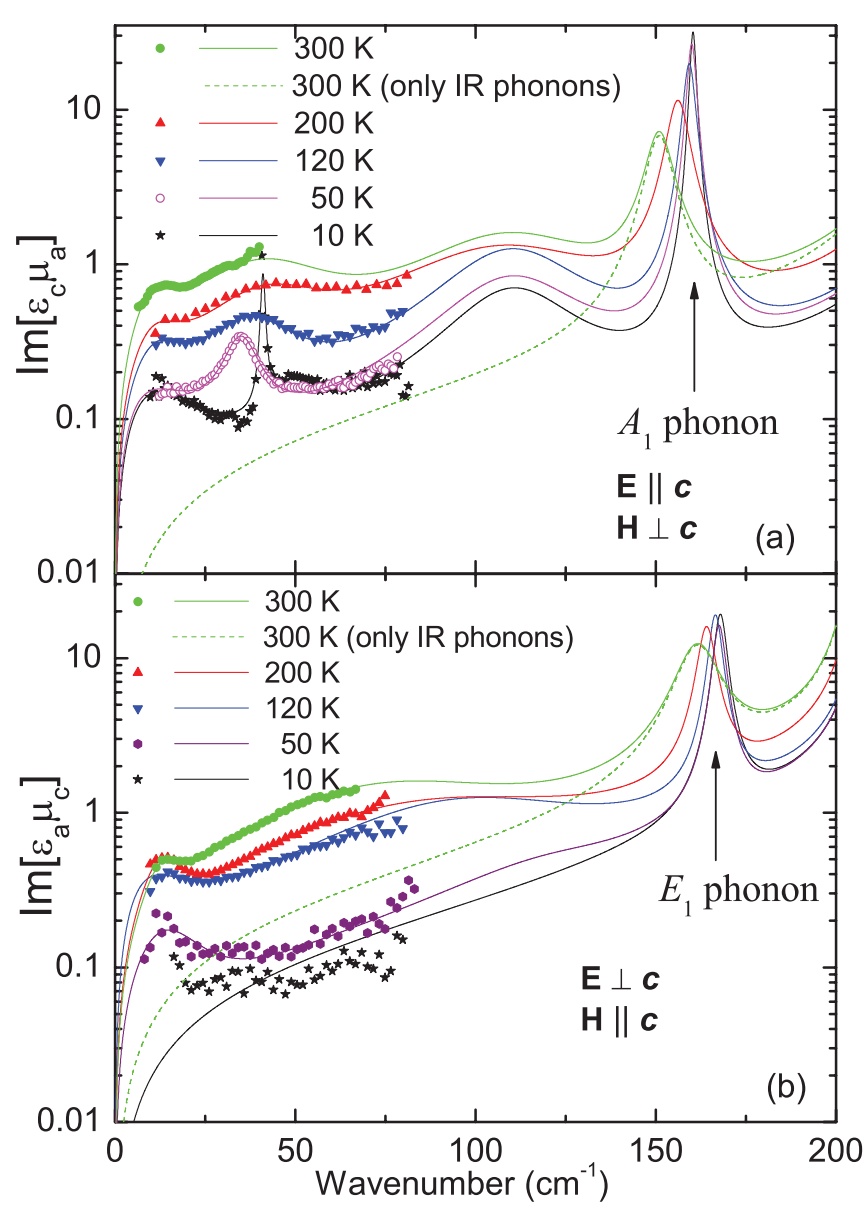

FIG. 5. (Color online) The measured THz loss spectra of $\mathrm{YMnO}_{3}$ (symbols) and those obtained from the fits of FTIR transmittance and reflectivity spectra. Below $T_{N}=70 \mathrm{~K}$, the spectra correspond to imaginary parts of the permittivity-permeability product. Above $T_{N}$, the spectra correspond to the dielectric losses. Polarizations of electric and magnetic components of IR or THz beams are indicated. Dashed lines are the fits of room-temperature FTIR reflectivity spectra without taking into account the IR and THz transmittance spectra. The marked peaks above $150 \mathrm{~cm}^{-1}$ are due to phonons; the origin of lower frequency absorption bands is discussed in the text.

spectral features (see Fig. 4). Their origin will be discussed in the next section.

The temperature dependence of the sub- $\mathrm{THz}$ complex dielectric permittivity $\varepsilon_{a}$ plotted in Fig. 6 for $20 \mathrm{~cm}^{-1}$ exhibits a pronounced drop below $T_{N}$. Such an anomaly is a typical feature of large spin-phonon coupling which occurs only in hexagonal planes of $\mathrm{YMnO}_{3}$, where the spins are ordered. For that reason the anomaly is not observed in $\varepsilon_{c}(T)$. The AFM phase transition is accompanied by unusually large atomic displacements, which were detected by neutron diffraction; ${ }^{28}$ for this reason the phonon frequencies change below $T_{N}$. The decrease in $\varepsilon_{a}^{\prime}$ and $\varepsilon_{a}^{\prime \prime}$ is mainly caused by hardening of the $E_{1}$ symmetry polar mode seen near $250 \mathrm{~cm}^{-1}$ in the IR reflectivity spectra with polarization $\mathbf{E} \perp \mathbf{c}^{30}$ Fits of our IR reflectivity spectra show that the mode near $250 \mathrm{~cm}^{-1}$ hardens from $246 \mathrm{~cm}^{-1}$ (at $300 \mathrm{~K}$ ) to $256 \mathrm{~cm}^{-1}$ (at $10 \mathrm{~K}$ ) and therefore its dielectric contribution $\Delta \varepsilon$ is reduced from $9.1(300 \mathrm{~K})$ to $7.6(10 \mathrm{~K})$. This decrease of $\Delta \varepsilon$ is mainly responsible for the 


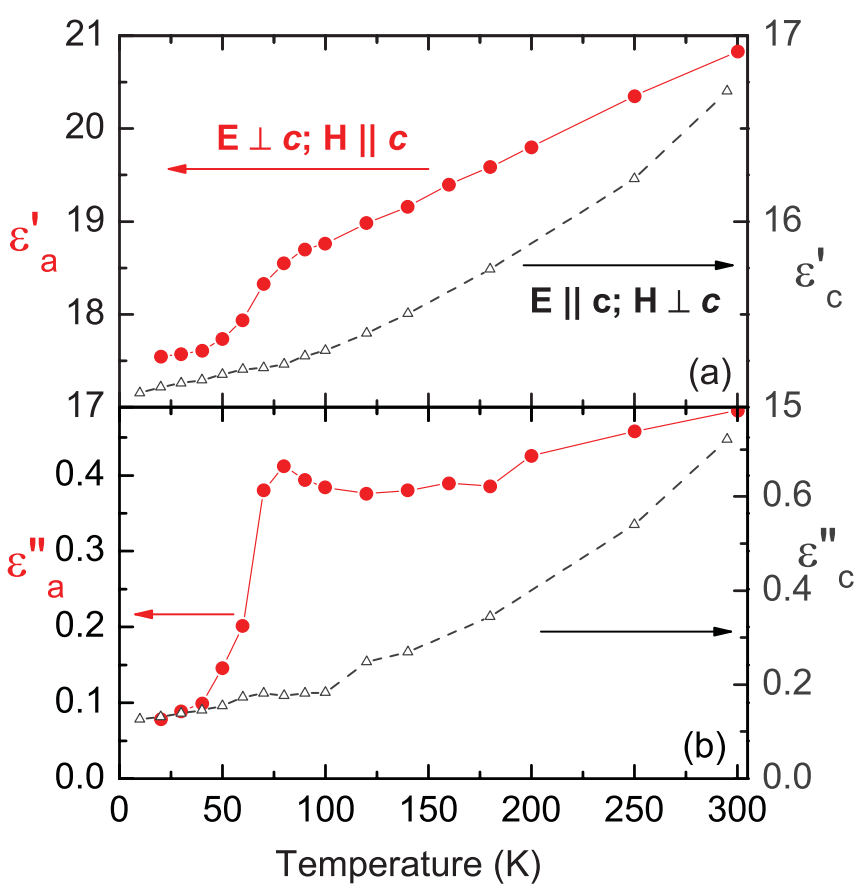

FIG. 6. (Color online) Temperature dependence of the (a) permittivity and (b) dielectric loss measured at $20 \mathrm{~cm}^{-1}$ with polarization $\mathbf{E} \perp \mathbf{c}$ (red solid lines) and $\mathbf{E} \| \mathbf{c}$ (black dashed lines).

change of the permittivity $\varepsilon_{a}^{\prime}(\mathrm{T})$ seen in Fig. 6. Hardening of other modes brings a minor contribution to the decrease of $\varepsilon_{a}^{\prime}(\mathrm{T})$ upon cooling. Similar temperature dependence of $\varepsilon_{a}^{\prime}$ was observed also in the radio-frequency region ${ }^{29}$ providing evidence of the absence of dielectric dispersion below $100 \mathrm{GHz}$. Gradual decrease of $\varepsilon_{a}^{\prime}$ and $\varepsilon_{c}^{\prime}$ upon cooling from 300 to $100 \mathrm{~K}$ is a usual behavior caused by a small phonon stiffening as a consequence of thermal contraction.

\section{DISCUSSION}

The question arises about the origin of the absorption bands appearing below phonon resonances in Fig. 5. They are much weaker and significantly broader than those of polar phonons, and their strength increases when the temperature is increased, i.e., the strength is high in the paramagnetic phase. Their frequencies lying in the range of $40-100 \mathrm{~cm}^{-1}$ coincide with those of the magnon branch observed by INS at $7 \mathrm{~K}$ over the $\mathrm{BZ}^{35}$ (see Fig. 7). In the following text we discuss whether these features can be related to the magnon dispersion branches.

Could a spin wave still exist in hexagonal $\mathrm{YMnO}_{3}$ at room temperature? It is well established that $\mathrm{Mn}$ spins exhibit a strong short-range correlation in hexagonal $\mathrm{YMnO}_{3}$ far above $T_{N}$. This was proved by an anomalous behavior of the thermal conductivity, ${ }^{44}$ elastic moduli, ${ }^{32}$ as well as by neutron scattering experiments. ${ }^{37-39}$ Nevertheless, due to the short-range correlation of the spins in the hexagonal plane of $\mathrm{YMnO}_{3}$, one can expect the existence of only short-wavelength paramagnons, i.e., magnons with large wave vectors $\boldsymbol{q}_{x}$ near the $M$ point of the BZ. A part of such a paramagnon branch is schematically plotted in Fig. 7. Note that its frequency is lower than that of the magnon branch at $7 \mathrm{~K}$, as the magnon

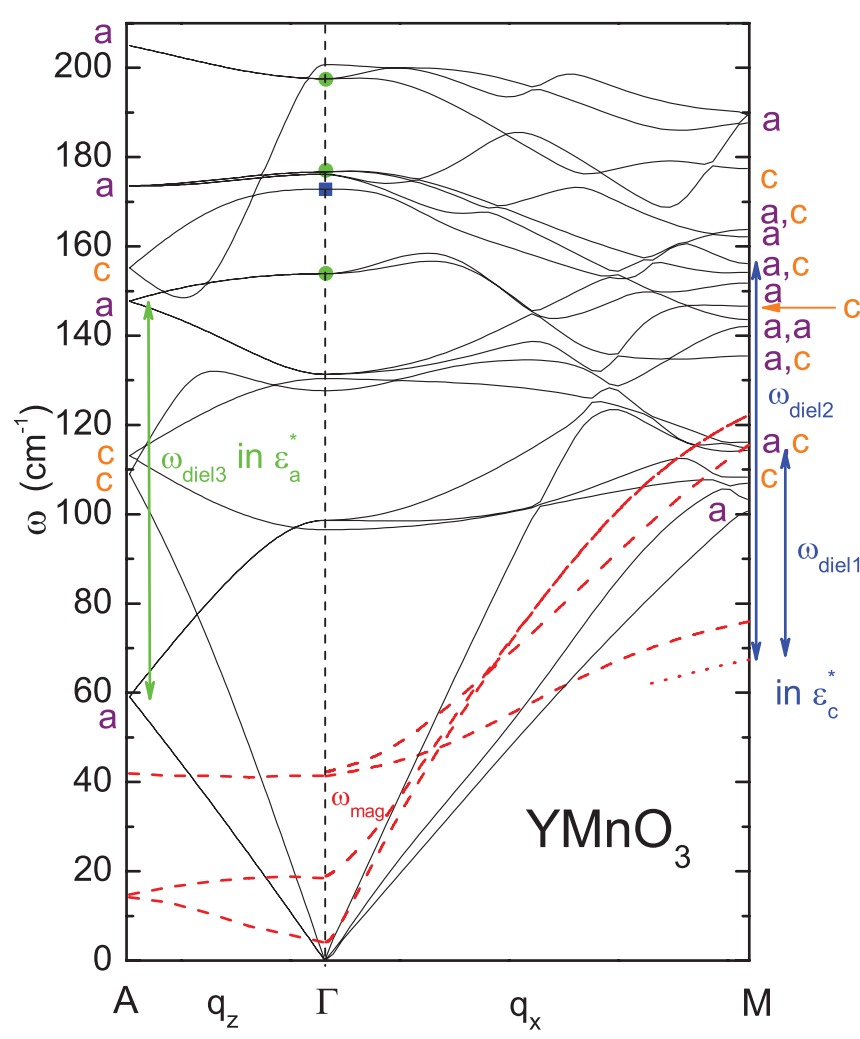

FIG. 7. (Color online) Dispersion branches of phonons (theoretical; black solid lines) and magnons (experimental ${ }^{35}$ at $7 \mathrm{~K}$; red dashed lines). The red-dotted line indicates the presumable dispersion of the paramagnon near the $M$ point. The symbols shown at the BZ edges indicate the polarization of the phonons at the BZ boundary: $a$ and $c$ stand for phonons polarized within the hexagonal plane and in the perpendicular direction, respectively. In the $\Gamma$-point, the $E_{1}$ and $A_{1}$ phonons observed experimentally ${ }^{30}$ are marked by green and blue points, respectively; other modes are silent. Blue arrows with assignment $\omega_{\text {diel1 }}$ and $\omega_{\text {diel } 2}$ indicate phonon-paramagnon excitations observed in the dielectric loss spectra of $\varepsilon_{c}^{\prime \prime}$. Green arrow marked as $\omega_{\text {diel3 }}$ indicates a broad multiphonon absorption observed in the $\varepsilon_{a}^{\prime \prime}$ loss spectra (see Fig. 5).

frequency decreases by almost $10 \mathrm{~cm}^{-1}$ on heating toward $T_{N}$ (see Fig. 2).

Electromagnons are excitations with frequencies close to those of spin waves, which, due to specific couplings, are activated in the dielectric spectra. In perovskite manganites, the parts of magnon branches exhibiting a high density of states are mainly involved in these interactions (at BZ edge or close to the spin modulation wave vector). ${ }^{13}$ However, these electromagnons were observed only at very low temperatures (typically less than $50 \mathrm{~K}$ ). Their strength dramatically decreases upon heating and they usually disappear from the spectra at $T_{N}$ or close above $T_{N} \cdot{ }^{4-6}$ This is in contradiction with our observations in $\mathrm{YMnO}_{3}$.

We came to the conclusion that the broad absorption bands we observe in the dielectric spectra reflect excitations which must be coupled to phonons. Let us discuss in brief which types of interaction between the magnetic subsystem and other degrees of freedom might be expected on the basis of the point group crystallographic symmetry $6 \mathrm{~mm}$ and the magnetic symmetry $\underline{6} \underline{m} \underline{m} .{ }^{21}$ The magnetic order parameter of 
$\mathrm{YMnO}_{3}$ was analyzed in several publications and it was shown to transform following $\mathrm{B}_{1}\left(\Gamma_{4}\right)$ irreducible representation of the $6 \mathrm{~mm}$ group. ${ }^{45-48}$ The $6 \mathrm{~mm}$ symmetry strictly forbids the linear magnetoelectric effect, i.e., bilinear terms $\alpha_{i j} H_{i} E_{j}$ (where $H_{i}$ and $E_{i}$ are components of the magnetic and electric field, respectively) are not allowed in the thermodynamic potential. $^{22}$ However, a higher order magnetoelectric effect (called sometimes the magnetodielectric effect), accounted for by the $\beta_{i j k} H_{i} H_{j} E_{k}$ terms in the thermodynamic potential, is allowed. This effect manifests itself in our measurements as a kink near $T_{N}$ in the temperature dependence of $\varepsilon_{a}^{\prime}$ (see Fig. 6).

The magnetic symmetry of $\mathrm{YMnO}_{3}$ allows the piezomagnetic contribution to the thermodynamic potential described by the terms $p_{i j k} H_{i} \sigma_{j k}$, where $\sigma_{j k}$ is a stress component and $p_{i j k}$ denotes components of the piezomagnetic tensor. ${ }^{22,24}$ We believe that this type of bilinear coupling must play an important role in the interaction between the magnetic subsystem and the lattice. Usually, the piezomagnetic effect is allowed thanks to the relativistic part of spin-lattice and spinspin interactions, provided the symmetry restrictions are met. ${ }^{25}$ However, in $\mathrm{YMnO}_{3}$, which is a noncollinear antiferromagnet, the exchange (Coulomb) interactions may be by several orders of magnitude stronger than the relativistic ones and, therefore, they can be the origin of piezomagnetism. ${ }^{49}$ For example, extraordinary spin-phonon interactions were shown to contribute to the thermal conductivity of $\mathrm{YMnO}_{3}$ below $T_{N} \cdot{ }^{44}$ Higher order effects such as $p_{i j k l} H_{i} H_{j} \sigma_{k l}$ are naturally also allowed in $\mathrm{YMnO}_{3}$.

In order to provide a more quantitative explanation of the interaction between magnetic subsystem and phonons, we calculated the phonon spectrum from first principles within the spin-polarized local density approximation. ${ }^{50} \mathrm{We}$ used projector augmented-wave potentials as implemented in Vienna $A b$ Initio Simulation Package (VASP). ${ }^{51-54}$ The following valence-electron configurations were considered: $4 s^{2} 4 p^{6} 5 s^{2} 4 d^{1}$ for $\mathrm{Y}, 3 p^{6} 4 s^{2} 3 d^{5}$ for $\mathrm{Mn}$, and $2 s^{2} 2 p^{4}$ for oxygen. To account for the strong electron correlation effects on the $d$ shells of Mn atoms, we used LDA $+U$ approach $^{55,56}$ with an on-site Coulomb parameter $U=8.0 \mathrm{eV}$ and Hund's exchange $J_{H}=0.88 \mathrm{eV}$ as calculated in Ref. 57. The spinorbit interaction was not taken into account. We used an $A$-type antiferromagnetic structure, where spins on Mn honeycomb layers are aligned ferromagnetically and the layers with opposite spin-direction alternate along the $c$ axis. ${ }^{58,59} \mathrm{~A}$ kinetic energy cutoff of $500 \mathrm{eV}$ and a $4 \times 4 \times 2 \Gamma$-centered $k$-point mesh was used in the structural relaxation of the unit cell, where the Hellman-Feynman forces were minimized to a value smaller than $0.5 \mathrm{meV} / \AA$. Phonon calculations were performed on a $2 \times 2 \times 1 \Gamma$-centered $k$-point mesh, with a $2 \times 2 \times 2$ supercell within the force-constant method. ${ }^{60,61}$ The Hellman-Feynman forces were calculated for displacements of atoms of up to $0.04 \AA$. The dynamical matrix for each $q$ point in the $\mathrm{BZ}$ was constructed by a Fourier transformation of the force constants, calculated for the $\Gamma$ point and for the BZ boundaries. Phonon-mode frequencies and atomic displacement patterns for each $q$ point were obtained as eigenvalues and eigenvectors of the dynamical matrices. The result for directions $A-\Gamma-M$ and wave numbers up to $200 \mathrm{~cm}^{-1}$ are presented in Fig. 7 .

As we have already pointed out, the absorption strength significantly increases upon heating. This is typical for difference frequency absorption. Such a process includes the annihilation of one quasiparticle (phonon or magnon) with frequency $\omega_{1}$ and the creation of another quasiparticle with a higher frequency $\omega_{2}$. The dielectric resonance then occurs at frequency $\omega_{\text {diel }}=\omega_{2}-\omega_{1}$. This process can involve excitations from the whole BZ provided that the total wave vector is conserved. The contribution of the parts of the dispersion branch with the highest density of states is expected to dominate. The high number of available states is found namely at the flat parts of the bands close to the BZ boundaries, as it was observed, for example, in $\mathrm{MgO}^{62}$

Obviously such a process is strongly temperature dependent, as it is related to the population of excitations with frequency $\omega_{1}$, which follows the Bose-Einstein statistics. At low temperatures, the population of the levels which we study is close to zero and the differential absorption then practically vanishes. It becomes more probable when the energy level is thermally populated at higher temperatures. This is in qualitative agreement with our observations.

The differential transitions at the BZ boundary are possible only between phonons with the same symmetry and if the total wave vector is conserved (i.e., the transition must be vertical in the wave-vector space). The broad absorption around $90 \mathrm{~cm}^{-1}$ seen in $\varepsilon_{a}$ spectra [Fig. 5(b)] can be explained by differential multiphonon absorption. Phonons near 60 and $150 \mathrm{~cm}^{-1}$ at the $A$ point of $\mathrm{BZ}$ are polarized in the hexagonal plane (marked as $a$ in Fig. 7) and their difference gives the frequency $\omega_{\text {diel } 3}=90 \mathrm{~cm}^{-1}$, as observed.

However, the two bands seen in $\varepsilon_{c}$ spectra around $\omega_{\text {diel1 }}=$ $40 \mathrm{~cm}^{-1}$ and $\omega_{\text {diel2 }}=100 \mathrm{~cm}^{-1}$ are impossible to explain by multiphonon absorption. The frequency of the $c$-polarized phonons at the BZ edge is higher than $100 \mathrm{~cm}^{-1}$. It means that the population of such phonons should be much lower than that of the $a$-polarized phonon at $60 \mathrm{~cm}^{-1}$. For this reason, the strength of the differential multiphonon absorption in the $\varepsilon_{c}$ spectra should be weaker than in $\varepsilon_{a}$ spectra. Moreover, within such a hypothesis, a continuous absorption band would be expected in the spectra due to the large number of $c$-polarized phonons at the $M$ point (see scheme in Fig. 7). This is in contradiction with the experimental results presented in Fig. 5.

We assume the existence of paramagnons near the $M$ point, and in this case a differential paramagnon-phonon absorption with several maxima can be obtained. Moreover, because of the similar Bose-Einstein factor for the paramagnon close to $70 \mathrm{~cm}^{-1}$ and phonon near $60 \mathrm{~cm}^{-1}$ at the $A$ point, the absorptions observed in both $\varepsilon_{a}$ and $\varepsilon_{c}$ should have comparable strengths. This fits well with the experiment. The frequency $\omega_{\text {diel1 }}$ increases upon heating (Fig. 3) presumably due to the softening of the paramagnon branch with increasing temperature. The increase of the oscillator strength $\Delta \varepsilon \omega_{\text {diel1 }}^{2}$ of the mode observed in Fig. 3 is compatible with the temperature increase of the Bose-Einstein factor: this is demonstrated by the dotted line which shows the expected population increase of an energy level at $66 \mathrm{~cm}^{-1}$ (i.e., the frequency of paramagnon at $\boldsymbol{q}_{\text {BZE }}$ ).

\section{CONCLUSIONS}

The THz and FTIR transmission spectra of hexagonal $\mathrm{YMnO}_{3}$ clearly revealed two kinds of excitations of different 
nature, which exist below polar phonon frequencies. The sharp AFM resonance band observed near $40 \mathrm{~cm}^{-1}$ at low temperatures broadens upon heating and disappears close to $T_{N}$. This resonance is the main contributor to the magnetic permeability $\mu_{a}$. Additional broad excitations were observed in the frequency range $40-100 \mathrm{~cm}^{-1}$ in the dielectric permittivity spectra in both the AFM and paramagnetic phases. Our theoretical explanation of the activation of these excitations in the $\mathrm{THz}$ dielectric spectra is based on two-particle differential processes schematically shown in Fig. 7. The resonance observed in $\varepsilon_{a}$ spectra is caused by differential phonon absorption in the $A$ point of the BZ. The two broad absorption bands in $\varepsilon_{c}$ spectra were described as differential phonon-paramagnon processes. The absorption strength of these excitations in the $\mathrm{THz}$ spectra increases upon heating due to the growing population of paramagnons and phonons with temperature. This is possible in the paramagnetic phase owing to strong short-range spin correlations within hexagonal planes of $\mathrm{YMnO}_{3}$. The processes we observe in $\mathrm{YMnO}_{3}$, where the linear magnetoelectric coupling is forbidden, are clearly different from the one responsible for the appearance of electromagnons in multiferroics with spin-induced ferroelectricity. ${ }^{1,2,4,5}$ The multiphonon absorptions are allowed by symmetry in all dielectric systems, while paramagnonphonon absorptions can be expected only in paramagnetic systems with a strong short-range magnetic order (e.g., in hexagonal manganites). Magnon-phonon absorption should be also detectable in all magnetically ordered systems (FM, AFM, ferrimagnets, etc.) with relatively high critical temperatures. In such conditions, the magnons at the Brillouin zone edge may become sufficiently populated to allow multiparticle effects in the spectra. This may stimulate further THz and FIR studies of other magnetically polarizable systems.

\section{ACKNOWLEDGMENTS}

The authors thank M. Mostovoy for valuable discussions. This work was supported by the Czech Science Foundation (Project No. 202/09/0682), by AVOZ10100520, and by the Young Investigators Group Program of the Helmholtz Association (Contract VH-NG-409). The contribution of Ph.D. student V.G. has been supported by Project Nos. 202/09/H041 and SVV-2011-263303. R.V.P. acknowledges the support by the RFBR (Project No. 09-02-00070). The support of the Jülich Supercomputing Center is gratefully acknowledged. *kamba@fzu.cz

${ }^{1}$ A. Pimenov, A. A. Mukhin, V. Yu. Ivanov, V. D. Travkin, A. M. Balbashov, and A. Loidl, Nature Phys. 2, 97 (2006).

${ }^{2}$ A. B. Sushkov, R. V. Aguilar, S. Park, S.-W. Cheong, and H. D. Drew, Phys. Rev. Lett. 98, 027202 (2007).

${ }^{3}$ V. G. Bar' yakhtar and I. E. Chupis, Sov. Phys. Solid State 11, 2628 (1970).

${ }^{4}$ A. Pimenov, A. M. Shuvaev, A. A. Mukhin, and A. Loidl, J. Phys. Condens. Matter 20, 434209 (2008).

${ }^{5}$ N. Kida, Y. Takahashi, J. S. Lee, R. Shimano, Y. Yamasaki, Y. Kaneko, S. Miyahara, N. Furukawa, T. Arima, and Y. Tokura, J. Opt. Soc. Am. B 26, A35 (2009).

${ }^{6}$ A. M. Shuvaev, A. A. Mukhin, and A. Pimenov, J. Phys. Condens. Matter 23, 113201 (2011).

${ }^{7}$ N. Kida, D. Okuyama, S. Ishiwata, Y. Taguchi, R. Shimano, K. Iwasa, T. Arima, and Y. Tokura, Phys. Rev. B 80, 220406(R) (2009).

${ }^{8}$ D. I. Khomskii, J. Magn. Magn. Mater. 306, 1 (2006).

${ }^{9}$ D. Khomskii, Physics 2, 20 (2009).

${ }^{10}$ Th. Lottermoser, D. Meier, R. V. Pisarev, and M. Fiebig, Phys. Rev. B 80, 100101 (2009).

${ }^{11}$ J. S. Lee, N. Kida, S. Miyahara, Y. Takahashi, Y. Yamasaki, R. Shimano, N. Furukawa, and Y. Tokura, Phys. Rev. B 79, 180403(R) (2009).

${ }^{12}$ P. Rovillain, M. Cazayous, Y. Gallais, M-A. Measson, A. Sacuto, H. Sakata, and M. Mochizuki, Phys. Rev. Lett. 107, 027202 (2011).

${ }^{13}$ R. Valdés Aguilar, M. Mostovoy, A. B. Sushkov, C. L. Zhang, Y. J. Choi, S.-W. Cheong, and H. D. Drew, Phys. Rev. Lett. 102, 047203 (2009).

${ }^{14}$ M. Mochizuki, N. Furukawa, and N. Nagaosa, Phys. Rev. Lett. 104, 177206 (2010).

${ }^{15}$ M. Cazayous, Y. Gallais, A. Sacuto, R. de Sousa, D. Lebeugle, and D. Colson, Phys. Rev. Lett. 101, 037601 (2008).
${ }^{16}$ G. Komandin, V. Torgashev, A. Volkov, O. Porodinkov, I. Spektor, and A. Bush, Phys. Solid State 52, 734 (2010).

${ }^{17}$ D. Talbayev, S. A. Trugman, S. Lee, H. T. Yi, S.-W. Cheong, and A. J. Taylor, Phys. Rev. B 83, 094403 (2011).

${ }^{18}$ A. S. Gibbs, K. S. Knight, and P. Lightfoot, Phys. Rev. B 83, 094111 (2011).

${ }^{19}$ E. Bertaut and M. Mercier, Phys. Lett. 5, 27 (1963).

${ }^{20}$ T. Chatterji, S. Ghosh, A. Singh, L. P. Regnault, and M. Rheinstädter, Phys. Rev. B 76, 144406 (2007).

${ }^{21}$ M. Fiebig, D. Fröhlich, K. Kohn, St. Leute, Th. Lottermoser, V. V. Pavlov, and R. V. Pisarev, Phys. Rev. Lett. 84, 5620 (2000).

${ }^{22}$ R. R. Birss, Symmetry and Magnetism (North-Holland, Amsterdam, 1967).

${ }^{23}$ M. Fiebig, Th. Lottermoser, D. Fröhlich, A. V. Goltsev, and R. V. Pisarev, Nature (London) 419, 818 (2002).

${ }^{24}$ A. V. Goltsev, R. V. Pisarev, Th. Lottermoser, and M. Fiebig, Phys. Rev. Lett. 90, 177204 (2003).

${ }^{25}$ L. D. Landau and E. M. Lifshitz, Electrodynamics of Continuos Media, 2ed. (Pergamon Press, Oxford, 1984).

${ }^{26}$ M. Fiebig, V. V. Pavlov, and R. V. Pisarev, J. Opt. Soc. Am. B 22, 96 (2005).

${ }^{27}$ T. Choi, Y. Horibe, H. T. Yi, Y. J. Choi, Wu Weida, and S.-W. Cheong, Nat. Mater. 9, 253 (2010).

${ }^{28}$ S. Lee, A. Pirogov, M. Kang, K.-H. Jang, M. Yonemura, T. Kamiyama, S.-W. Cheong, F. Gozzo, N. Shin, H. Kimura, Y. Noda, and J.-G. Park, Nature (London) 451, 805 (2008).

${ }^{29}$ Y. Aikawa, T. Katsufuji, T. Arima, and K. Kato, Phys. Rev. B 71, 184418 (2005)

${ }^{30}$ M. Zaghrioui, V. Ta Phuoc, R. A. Souza, and M. Gervais, Phys. Rev. B 78, 184305 (2008).

${ }^{31}$ H. Fukumura, S. Matsui, H. Harima, K. Kisoda, T. Takahashi, T. Yoshimura, and N. Fujimura, J. Phys. Condens. Matter 19, 365239 (2007). 
${ }^{32}$ M. Poirier, F. Laliberté, and L. Pinsard, and A. Revcolevschi, Phys. Rev. B 76, 174426 (2007).

${ }^{33}$ T. Penney, P. Berger, and K. Kritiyakirana, J. Appl. Phys. 40, 1234 (1969).

${ }^{34}$ V. Goian, S. Kamba, C. Kadlec, D. Nuzhnyy, P. Kužel, J. Agostino Moreira, A. Almeida, and P. B. Tavares, Phase Transitions 83, 931 (2010).

${ }^{35}$ T. J. Sato, S.-H. Lee, T. Katsufuji, M. Masaki, S. Park, J. R. D. Copley, and H. Takagi, Phys. Rev. B 68, 014432 (2003).

${ }^{36}$ S. Petit, F. Moussa, M. Hennion, S. Pailhès, L. Pinsard-Gaudart, and A. Ivanov, Phys. Rev. Lett. 99, 266604 (2007).

${ }^{37}$ J. Park, J.-G. Park, G. S. Jeon, H.-Y. Choi, Ch. Lee, W. Jo, R. Bewley, K. A. McEwen, and T. G. Perring, Phys. Rev. B 68, 104426 (2003).

${ }^{38}$ B. Roessli, S. N. Gvasaliya, E. Pomjakushina, and K. Conder, JETP Lett. 51, 287 (2005).

${ }^{39}$ F. Demmel and T. Chatterji, Phys. Rev. B 76, 212402 (2007).

${ }^{40} \mathrm{~S}$. Pailhès, X. Fabrèges, L. P. Régnault, L. Pinsard-Godart, I. Mirebeau, F. Moussa, M. Hennion, and S. Petit, Phys. Rev. B 79, 134409 (2009).

${ }^{41}$ P. Kužel, H. Němec, F. Kadlec, and C. Kadlec, Opt. Express 18, 15338 (2010).

${ }^{42}$ S. L. Dexheimer, $\mathrm{THz}$ Spectroscopy: Principles and Applications (CRC Press, Boca Raton, FL, 2008).

${ }^{43}$ H. Yamagichi, T. Fujita, T. Shinozaki, H. Sigie, and K. Kohn, Ferrites: Proceedings of the Eighth International Conference on Ferrites (ICF 8), Kyoto and Tokyo, Japan 2000 .
${ }^{44}$ P. A. Sharma, J. S. Ahn, N. Hur, S. Park, S. B. Kim, S. Lee, J.-G. Park, S. Guha, and S.-W. Cheong, Phys. Rev. Lett. 93, 177202 (2004).

${ }^{45}$ G. M. Nedlin, Sov. Phys. Solid State 6, 2156 (1965).

${ }^{46}$ Yu. G. Pashkevich, V. L. Sobolev, S. A. Fedorov, and A. V. Eremenko, Phys. Rev. B 51, 15898 (1995).

${ }^{47}$ D. Sa, R. Valentí, and C. Gros, Eur. Phys. J. B 14, 301 (2000).

${ }^{48}$ G. F. Koster, J. O. Dimmock, R. G. Wheeler, and H. Statz, Properties of the Thirty Two Point Groups (MIT Press, Cambridge, 1963).

${ }^{49}$ I. M. Vitebskii, N. M. Lavrinenko, and V. L. Sobolev, J. Magn. Magn. Mater. 97, 263 (1991).

${ }^{50}$ J. P. Perdew and A. Zunger, Phys. Rev. B 23, 5048 (1981).

${ }^{51}$ G. Kresse and J. Hafner, Phys. Rev. B 47, 558 (1993).

${ }^{52}$ G. Kresse and J. Furthmüller, Phys. Rev. B 54, 11169 (1996).

${ }^{53}$ P. E. Blöchl, Phys. Rev. B 50, 17953 (1994).

${ }^{54}$ G. Kresse and D. Joubert, Phys. Rev. B 59, 1758 (1999).

${ }^{55}$ V. I. Anisimov, F. Aryasetiawan, and A. I. Lichtenstein, J. Phys. Condens. Matter 9, 767 (1997).

${ }^{56}$ S. L. Dudarev, G. A. Botton, S. Y. Savrasov, C. J. Humphreys, and A. P. Sutton, Phys. Rev. B 57, 1505 (1998).

${ }^{57}$ J. E. Medvedeva, V. I. Anisimov, M. A. Korotin, O. N. Mryasov, and A. J. Freeman, J. Phys. Condens. Matter 12, 4947 (2000).

${ }^{58}$ C. J. Fennie and K. M. Rabe, Phys. Rev. B 72, 100103(R) (2005).

${ }^{59}$ B. B. van Aken, T. T. M. Palstra, A. Filippetti, and N. A. Spaldin, Nat. Mater. 3, 164 (2004).

${ }^{60}$ D. Alfè, Comput. Phys. Commun. 180, 2622 (2009).

${ }^{61}$ K. Kunc and R. M. Martin, Phys. Rev. Lett. 48, 406 (1982).

${ }^{62}$ G. A. Komandin, O. E. Porodinkov, I. E. Spector, and A. A. Volkov, Phys. Solid State 51, 2045 (2009). 